\title{
DYNAMIC ROUTING OF CUSTOMERS WITH GENERAL DELAY COSTS IN A MULTISERVER QUEUING SYSTEM
}

\author{
Nilay TANIK Argon \\ Department of Statistics and Operations Research \\ University of North Carolina at Chapel Hill \\ Chapel Hill, NC 27599 \\ E-mail:nilay@unc.edu \\ LI DING \\ Durham Business School \\ Durham University \\ Durham, DH1 3LB, UK \\ E-mail: li.ding@durham.ac.uk \\ Kevin D. Glazebrook \\ Department of Mathematics and Statistics \\ Lancaster University Management School \\ Bailrigg, Lancaster LA1 4YX, UK \\ E-mail:k.glazebrook@lancaster.ac.uk \\ SERHAN ZiYA \\ Department of Statistics and Operations Research \\ University of North Carolina at Chapel Hill \\ Chapel Hill, NC 27599 \\ E-mail: ziya@unc.edu
}

\begin{abstract}
We consider a network of parallel service stations each modeled as a single-server queue. Each station serves its own dedicated customers as well as generic customers who are routed from a central controller. We suppose that the cost incurred by a customer is an increasing function of her time spent in the system. In a significant advance on most previous work, we do not require waiting costs to be convex, still less linear. With the objective of minimizing the long-run average waiting cost, we develop two heuristic routing policies, one of which is based on dynamic programming policy improvement and the other on Lagrangian relaxation. In developing the
\end{abstract}


latter policy, we show that each station is "indexable" under mild conditions for customers' waiting costs and also prove some structural results on the admission control problem that naturally arises as a result of the Lagrangian relaxation. We then test the performance of our heuristics in an extensive numerical study and show that the Lagrangian heuristic demonstrates a strong level of performance in a range of traffic conditions. In particular, it clearly outperforms both a greedy heuristic, which is a standard proposal in complex routing problems, and a recent proposal from the heavy traffic literature.

\section{INTRODUCTION}

This article is concerned with the dynamic routing of incoming customers to one of several parallel service stations, each of which has its own queue with an infinite buffer space. Our model develops those found most frequently in the literature in two key respects, both of which are critical for applications. First, in our setups there are two customer types that inhabit the system: dedicated and generic. Dedicated customers must be served at a designated station and routing decisions only concern the remaining generic traffic, which we will assume can be served at any station. Plainly, such a dichotomizing of the traffic is important in settings where specialist skills are required on occasion and are expensive to provide. Note that stations are not necessarily separated geographically since customers requiring different skills will naturally form separate queues. Examples include call centers [7] and emergency response systems [31]. Such models are also relevant to dynamic routing within a grid environment [1] and to load balancing in broadcast communication networks [37].

Both customer types incur costs that depend on the time spent waiting in the system. Our objective is to develop routing policies that will minimize the long-run average total cost or that will come close to doing so. This brings us to the second key feature of our model, namely that we will impose only very minor conditions on the structure for waiting costs. Most previous work that has considered the dynamic service and routing control of queuing systems has imposed requirements that waiting costs be linear in the time spent in system. In some exceptions, this has been generalized to an assumption that costs be convex nondecreasing (see, e.g., $[14,34,40,45])$. Van Mieghem [45] discussed the limited appeal of linear waiting costs, but we would argue that an assumption of convexity might also be unrealistic in many settings. Convex waiting costs are unable to take an adequate account of such commonplace features as delivery time/service-level guarantees (see, e.g., [10,20,24]) or in an emergency response setting of the absolute imperative of treating patients with critical trauma injuries quickly (the "golden hour" rule; see, e.g., [35]).

State-dependent routing problems have received much attention in the literature. The only general settings in which it has proved possible to elucidate simple structure for optimal policies are those involving homogeneous stations. Here, "Shortest Delay Routing" (SDR) has been shown to be optimal under a range of conditions. See, 
for example, Weber [47], Winston [52], Johri [23], Hordijk and Koole [21], Menich and Serfozo [33], Sparaggis, Towsley, and Cassandras [39], Towsley, Sparaggis, and Cassandras [44], and Koole, Sparaggis, and Towsley [26]. However, Whitt [49] showed that SDR might not be optimal even for very simple cases. See Foschini and Salz [12], Houck [22], Wein [48], Laws [30], Kelly and Laws [25], Foley and McDonald [11], Teh and Ward [41], and Tezcan [42] for more on the performance of SDR and analytical results for SDR under various asymptotic regimes. Recently, there also appeared a significant amount of work on routing problems in which customers wait in a single line and are routed to one of the servers as servers become available. The majority of this work is motivated by the routing problem in call centers. See, for example, Van Mieghem [45], Gans and Zhou [13], Harrison and Zeevi [19], Mandelbaum and Stolyar [32], Armony and Maglaras [4,5], Wallace and Whitt [46], Armony [3], and Bassamboo, Harrison, and Zeevi [6].

In a recent study, Stolyar [40] considered a similar routing problem in which customers are routed to one of the multiple parallel stations as they arrive to the system. Stolyar's model is more general than ours in that customers belong to different classes and service rates depend on both the customer's class identity and the station serving the customer. However, the author imposed some restrictive conditions on the waiting cost structures. More specifically, he assumed that the waiting cost function for each queue is continuous, strictly increasing, and convex. Stolyar proved that a routing policy, which he calls the MinDrift rule, is asymptotically optimal in the heavy traffic regime under a complete resource pooling condition. In Section 6, we test the performance of the MinDrift rule along with the performance of our heuristics under various traffic regimes and two different waiting cost functions.

For complex routing problems that do not assume station homogeneity, full characterization of optimal policies based, for example, on direct application of dynamic programming (DP) methodology is unrealistic. The focus for such systems has mostly been on the development of effective routing heuristics, many of which are based on calibrations of the constituent service stations that utilize queue length information. A simple and standard proposal is to route each generic customer in an individually optimal way to whichever station has the smallest expected cost for that individual. In Section 6, this is referred to as the greedy heuristic (GH). Two approaches to heuristic development which have proved effective are those based on DP policy improvement (see, e.g., Krishnan and Ott [29], Krishnan [27,28], Whittle [50], Sassen, Tijms, and Nobel [38], Ansell, Glazebrook, and Kirkbride [1], Bhulai and Koole [9], and Bhulai [8]) and on Lagrangian relaxations (see, for example, Niño-Mora [34] and Whittle [50,51]). The second of these has appeared highly promising, but its effective deployment has been severely inhibited by a prior requirement to establish a structural property which Whittle [51] called indexability. While this property appears natural, it is little understood and is often extremely difficult to prove. There is a developing literature centered around this very issue in a range of application areas of which routing is but one (see, e.g., Glazebrook, Niño-Mora, and Ansell [17], Ansell, Glazebrook, Niño-Mora, and O'Keeffe [2], Glazebrook, Mitchell, and Ansell [16], and Glazebrook, Kirkbride, and Ouenniche [15]). 
A major research achievement of this article is the demonstration that we do indeed have indexability in our very general setting for both costs and traffic. In particular, we are able to show that the cost convexity assumptions of previous work are not needed for the parallel service system considered in this article. Indexability is proved in Section 4 and the structure of the resulting routing heuristics is established. To provide a strong comparator for these Lagrangian heuristics, routing policies based on DP policy improvement are developed in Section 3. In Section 5, the precise nature of the station calibrations that our routing heuristics utilize is developed for some nonstandard but practically important cost structures and Section 6 contains the results of an extensive investigation into heuristic performance. Finally, Section 7 provides our concluding remarks and directions for future research.

\section{MODEL DESCRIPTION}

We consider $N \geq 2$ parallel service stations, each modeled as a single-server queue. For each queue $n$, service times of customers are independent and exponentially distributed with a finite mean $1 / \mu_{n}$. Each queue $n$ has its own "dedicated" customers that arrive according to a Poisson process with rate $\eta_{n}$. These dedicated customers cannot be routed to any other station. There are also "generic" customers who arrive as a Poisson stream with rate $\lambda$ and who can be served at any station. The routing decisions concern these customers only. Our analysis will still go through if the system controller can also choose to reject generic customers by paying a fixed charge per rejected customer. However, to keep the presentation simpler, we assume that each arriving customer should be admitted to the system. The routing decision for a generic customer is made upon her arrival and this decision is irreversible. We assume that each queue operates under a first-come-first served (FCFS) discipline; hence, no distinction is made between the dedicated customers and generic customers once admitted to the system. We introduce the parameters

$$
\theta_{n}=\lambda+\eta_{n}, \quad \beta_{n}=\theta_{n} / \mu_{n}, \quad \alpha_{n}=\eta_{n} / \mu_{n},
$$

and we assume that $\alpha_{n}<1$ for all $n$ and $\lambda+\sum_{n=1}^{N} \eta_{n}<\sum_{n=1}^{N} \mu_{n}$ so that there exist routing policies under which the system is stable.

Each customer incurs a nonnegative cost that is a function of the time the customer spends in the system. Write $\xi_{n k}$ for $n \in\{1,2, \ldots, N\}$ and $k \in\{1,2, \ldots\}$ for the total system time for the $k$ th customer admitted to queue $n$. Also, let $C_{n}(\cdot)$ denote the cost function for queue $n$; that is, $C_{n}(t)$ is the cost incurred by a customer who is given service in station $n$ and whose total system time is $t$. Our objective is to minimize the expected long-run average total cost for this system, which can be written as

$$
\limsup _{t \rightarrow \infty} \frac{\mathbb{E}\left[\sum_{n=1}^{N} \sum_{k=1}^{Z_{n}(t)} C_{n}\left(\xi_{n k}\right)\right]}{t}
$$


where $Z_{n}(t)$ is the total number of customers who have joined station $n$ by time $t$. Note that since the above expression uses a convention that the total waiting cost for each customer is incurred as soon as the customer joins, the numerator is in fact an upper bound on the total expected costs accumulated by time $t$. However, for any policy under which the number in system remains finite almost surely, for any given $t$, the difference between this upper bound and the actual value of the accumulated costs will be finite with probability 1 so that as time $t$ converges to infinity, the difference between the actual cost rate and its upper bound converges to zero.

Let $c_{n}(i)$ denote the expected cost that will be incurred by a customer who joins queue $n$ when there are $i$ customers already waiting (including the customer in service) at station $n$. If we deem that (expected) costs are incurred as the customers join, this problem can be formulated as a semi-Markov decision process. Decision epochs are the times when generic customers arrive and the system state can be described by an $N$-dimensional vector $\boldsymbol{x}=\left(x_{1}, x_{2}, \ldots, x_{N}\right)$, where $x_{n}$ denotes the number of customers waiting in station $n$ (including the customer in service). The action space is $\mathcal{A}=\left\{a_{1}, a_{2}, \ldots, a_{N}\right\}$, where $a_{n}$ is the action of sending a customer to queue $n$. Once the problem is formulated, then it can, in principle, be solved to arbitrary accuracy by applying a (finite) truncation of the state space followed by deployment of standard DP methodologies. However, such an approach is computationally intractable for systems of realistic size. It is natural that interest should focus on the development of effective heuristics for routing. This is the subject of the following two sections.

\section{HEURISTIC 1: SINGLE-STEP POLICY IMPROVEMENT METHOD}

We develop the single-step policy improvement heuristic by applying a single step of the policy improvement algorithm to an optimal static Bernoulli routing policy. A static Bernoulli routing policy simply routes each incoming customer to queue $n$ with some probability $p_{n}$ independently of other customers and the system state. We will develop the heuristic in two stages. First, we will determine an optimal static Bernoulli routing policy. This will then be followed by a single DP policy improvement step.

\subsection{An Optimal Static Policy}

A static routing policy routes each incoming generic customer to one of the service stations according to some probability distribution $\boldsymbol{p}=\left(p_{1}, p_{2}, \ldots, p_{N}\right)$ independently of other customers and the system state, where $p_{n} \geq 0$ for $n=1,2, \ldots, N$ and $\sum_{n=1}^{N} p_{n}=1$. Let the set $\mathbb{P}$ be defined as

$$
\mathbb{P}=\left\{\boldsymbol{p}: p_{n} \geq 0, \eta_{n}+\lambda p_{n}<\mu_{n}, 1 \leq n \leq N, \text { and } \sum_{n=1}^{N} p_{n}=1\right\} .
$$

We have $\mathbb{P} \neq \emptyset$ since we assume that $\lambda+\sum_{n} \eta_{n}<\sum_{n} \mu_{n}$. 
Under any static policy $\boldsymbol{p} \in \mathbb{P}$, each service station $n$ is an independent $M / M / 1$ queue with arrival rate $\Lambda_{n}=\eta_{n}+\lambda p_{n}$ and service rate $\mu_{n}$. Then, using standard results, the expected long-run average cost $T C(\boldsymbol{p})$, can be written as

$$
T C(\boldsymbol{p})=\sum_{n=1}^{N}\left(\eta_{n}+\lambda p_{n}\right) \sum_{i=0}^{\infty} c_{n}(i)\left(1-\rho_{n}\right) \rho_{n}^{i},
$$

where $\rho_{n}=\left(\eta_{n}+\lambda p_{n}\right) / \mu_{n}$.

In order to proceed, we need the following assumption:

Assumption 3.1: There exists a $\boldsymbol{p} \in \mathbb{P}$ such that $\sum_{n=1}^{N} \sum_{i=0}^{\infty} c_{n}(i) \rho_{n}^{i}<\infty$, where $\rho_{n}=\left(\eta_{n}+\lambda p_{n}\right) / \mu_{n}$.

Assumption 3.1 is certainly satisfied if the $c_{n}(\cdot)$ 's are all polynomially bounded.

It is easy to see that as $\mathbf{p}$ converges to one of the boundary points of $\mathbb{P}, T C(\mathbf{p})$ diverges. Furthermore, under Assumption 3.1, there exists a static routing policy $\mathbf{p} \in \mathbb{P}$ such that $T C(\mathbf{p})$ is finite. These show that a minimizer for $T C(\mathbf{p})$ is contained in $\mathbb{P}$. Hence, we can define an optimal static routing policy as any vector $\boldsymbol{p}^{*} \in \mathbb{P}$ that minimizes (1); that is,

$$
\boldsymbol{p}^{*} \in\{\overline{\boldsymbol{p}} \in \mathbb{P}: T C(\overline{\boldsymbol{p}}) \leq T C(\boldsymbol{p}) \quad \forall \boldsymbol{p} \in \mathbb{P}\} .
$$

\subsection{Policy Improvement Step}

We now apply a policy improvement step to an optimal static policy. Using the approach of Section 3.6 of Tijms [43], it can be easily shown that the policy improvement step yields an index policy. To see that, we first define $\Delta\left(\boldsymbol{x}, a_{n}\right)$ to be the difference in total expected costs that would be caused by sending the first incoming customer to queue $n$ and then following an optimal static policy rather than using an optimal static policy throughout when the system is in state $\boldsymbol{x}$. Since each queue operates independently under a static policy, this difference can be expressed as

$$
\begin{aligned}
\Delta\left(\boldsymbol{x}, a_{n}\right) & =\sum_{k=1}^{N} p_{k}^{*}\left[D_{n}\left(x_{n}\right)-D_{k}\left(x_{k}\right)\right] \\
& =D_{n}\left(x_{n}\right)-\sum_{k=1}^{N} p_{k}^{*} D_{k}\left(x_{k}\right),
\end{aligned}
$$

where $D_{n}(i)$ denotes the additional long-run cost of admitting an extra customer to queue $n$ operating under the optimal static routing policy when $i$ customers are already present. In (3), the summation term does not depend on the action $a_{n}$ taken. Therefore, the action $a_{n}$ that minimizes $\Delta\left(\boldsymbol{x}, a_{n}\right)$ can simply be found by choosing $n$ to minimize 
$D_{n}\left(x_{n}\right)$. In other words, the policy improvement step gives us an index policy that routes an arriving generic customer to queue $n^{*}$ such that

$$
D_{n^{*}}\left(x_{n^{*}}\right)=\min _{n \in\{1,2, \ldots, N\}}\left\{D_{n}\left(x_{n}\right)\right\}
$$

when the system state is $\boldsymbol{x}=\left(x_{1}, x_{2}, \ldots, x_{N}\right)$.

In order to describe the policy, we require an expression for $D_{n}(\cdot)$. It will simplify matters if we now focus on a single station and drop the identifier $n$. Let $\Lambda=\eta+\lambda p^{*}$ denote the total arrival rate to this station (the total arrival rate of dedicated and generic customers assigned to this queue under the optimal static policy). Define $\mu$ to be the service rate, $\rho=\Lambda / \mu$ to be the traffic intensity, where $\rho<1$, and $c(i)$ to be the expected cost for a customer who sees $i$ customers upon arrival.

Utilizing standard arguments that exploit the fact that the system regenerates upon every return to the empty state, it can be shown that

$$
D(i)=c(i)+\left(K_{i+1}-g T_{i+1}\right)-\left(K_{i}-g T_{i}\right) \quad \text { for } i \geq 0,
$$

where $K_{i}$ is the expected total cost incurred until the first time there is no customer in the system starting with $i$ customers, $T_{i}$ is the expected time until the first time the station is empty starting with $i$ customers, and $g$ is the long-run average cost for the queue under the static policy. Note that $K_{0}=0$ and $T_{0}=0$.

To make sense of (4), note that $K_{i}-g T_{i}$ is the relative cost (or bias) of starting the queue with $i$ customers rather than none. Hence, the difference between the last two terms in (4) is simply the difference in total expected long-run costs between starting the queue with $i+1$ rather than $i$ customers. However, this difference ignores the cost to be incurred by the customer to be admitted. Adding the expected cost for that customer, $c(i)$, to this difference gives us the additional cost of admitting one more customer, which is $D(i)$. Now, we can rewrite (4) as follows:

$$
D(i)=c(i)+K_{i+1}-K_{i}+g\left(T_{i}-T_{i+1}\right) \quad \text { for } i \geq 0 .
$$

To find an expression for $K_{i}$, we first note that

$$
K_{i}=\frac{\Lambda}{\Lambda+\mu}\left(c(i)+K_{i+1}\right)+\frac{\mu}{\Lambda+\mu} K_{i-1} \text { for } i \geq 1 .
$$

Rearranging terms, we obtain

$$
k_{i+1}=\rho^{-1} k_{i}-c(i),
$$

where $k_{i}=K_{i}-K_{i-1}$. Using this relationship recursively, we get

$$
k_{i}=\frac{k_{1}-\sum_{j=1}^{i-1} c(j) \rho^{j}}{\rho^{i-1}} \quad \text { for } i \geq 2 .
$$

Note that $k_{1}=K_{1}-K_{0}=K_{1}$. Hence, to give an explicit expression for $k_{i}$, it remains to determine $K_{1}$. 
In order to derive $K_{1}$, consider the $M / M / 1$ queue under study. This queue evolves in cycles [i.e., independent and identically distributed (i.i.d.) renewal intervals], which start when a customer arrives to an empty system. Each cycle consists of a busy period (there are customers in the system and the server is working) followed by an idle period (no customers in the system and therefore the server is idle). Let $\gamma_{m}$ denote the total cost incurred over cycle $m$. Clearly, $\gamma_{m}$ is i.i.d. for all $m$. Let $\Gamma=E\left[\gamma_{1}\right]$.

Recall that $g$ is the long-run average cost for this system. Then using the renewal reward theorem and the fact that expected length of a busy period is $1 /(\mu-\Lambda)$ (see, e.g., [18, Sect. 2.11]), we can show the following:

$$
\Gamma=\frac{g}{\Lambda(1-\rho)} .
$$

Then

$$
K_{1}=\Gamma-c(0)
$$

since $K_{1}$ does not include expected costs for the customer initially in the system. Then, using (6) and the fact that $g=\Lambda \sum_{j=0}^{\infty} c(j)(1-\rho) \rho^{j}$, after a few algebraic manipulations, we can establish that

$$
k_{i}=\frac{\sum_{j=i}^{\infty} c(j) \rho^{j}}{\rho^{i-1}} \quad \text { for } i \geq 1 .
$$

Finally, since $T_{i+1}-T_{i}$ is equal to the expected length of a "busy period," we have $T_{i+1}-T_{i}=1 /(\mu-\Lambda)$. Then, using (7), we deduce that

$$
D(i)=c(i)+k_{i+1}-g \frac{1}{\mu-\Lambda}=\sum_{j=0}^{\infty}(c(j+i)-\rho c(j)) \rho^{j}
$$

for $i \geq 0$. \{An alternative way of obtaining (8) from (4) is by solving for the relative values $K_{i}-g T_{i}$ directly, utilizing the form of solution to classes of second-order difference equations given as Corollary 3.3 in Bhulai [8].\}

Thus, we have proved the following theorem.

THEOREM 3.1 (Policy Improvement Heuristic): The heuristic dynamic routing policy that is developed by applying a policy improvement step to an optimal static policy $\boldsymbol{p}^{*}=\left(p_{1}^{*}, p_{2}^{*}, \ldots, p_{N}^{*}\right)$ operates as follows: Upon arrival of a generic customer, if the system state is $\boldsymbol{x}=\left(x_{1}, x_{2}, \ldots, x_{n}\right)$, then route the customer to any station $n^{*}$ for which

$$
D_{n^{*}}\left(x_{n^{*}}\right)=\min _{k \in\{1,2, \ldots, N\}}\left\{D_{k}\left(x_{k}\right)\right\}
$$

where

$$
D_{k}\left(x_{k}\right)=\sum_{j=0}^{\infty}\left(c_{k}\left(j+x_{k}\right)-\rho_{k} c_{k}(j)\right) \rho_{k}^{j}
$$

and $\rho_{k}=\left(\eta_{k}+\lambda p_{k}^{*}\right) / \mu_{k}$. 
The index function (9) is insightful. To see that, we can use (7) and (8) to rewrite (9) as

$$
\begin{aligned}
D_{k}\left(x_{k}\right)= & c_{k}\left(x_{k}\right)+\frac{1}{\mu_{k}-\Lambda_{k}}\left(\frac{\Lambda_{k}}{\rho_{k}^{x_{k}+1}} \sum_{i=x_{k}+1}^{\infty} c_{k}(i) \rho_{k}^{i}\left(1-\rho_{k}\right)\right. \\
& \left.-\Lambda_{k} \sum_{i=0}^{\infty} c_{k}(i) \rho_{k}^{i}\left(1-\rho_{k}\right)\right) .
\end{aligned}
$$

The first term on the right-hand side of $(10), c_{k}\left(x_{k}\right)$, is the expected cost for a customer who will be sent to station $k$ and might be interpreted as the internal cost for the customer. However, the customer also incurs costs for the other incoming customers who will be routed to the same station and the second term of (10) takes this cost into account. The term $1 /\left(\mu_{k}-\Lambda_{k}\right)$ is the expected length of a "busy period" caused by the customer under the optimal static policy. In other words, it is the expected time until the first occasion for which the number of customers in the station is $x_{k}$ again. The first term in the parentheses in (10) is the long-run average cost for the station when the average is taken over the times when there are more than $x_{k}$ customers in the station under the optimal static policy, whereas the second term in the parentheses is simply the long-run average cost for station $k$. Their difference can be interpreted as the per unit time cost of keeping the queue above level $x_{k}$. Multiplying this difference by the expected length of a busy period, we find the total cost that the admitted customer imposes on the system. Hence, in a sense, the expression that follows $c_{k}\left(x_{k}\right)$ in (10) is the external cost of admitting the customer to station $k$.

\section{HEURISTIC 2: LAGRANGIAN RELAXATION METHOD}

In order to describe the development of an alternative collection of routing heuristics, we first reexpress the optimization problem of interest as

$$
C^{o p t}=\min _{u \in \mathcal{U}} \sum_{n=1}^{N} \widetilde{C}_{n}(u),
$$

where $\mathcal{U}$ is the class of stationary policies for routing generic arrivals and $\widetilde{C}_{n}(u)$ is the time-average cost rate incurred at station $n$ under control $u \in \mathcal{U}$.

We now follow Whittle's [51] approach to the analysis of the intractable class of restless bandit problems by relaxing the problem in such a way that the (hard) constraint that each generic arrival be routed to exactly one station is replaced by the (softer) constraint that generic arrivals be routed to one station on average. Expressed differently, the class of policies is expanded to those that route each incoming generic customer to any number of service stations, subject to an overall admission rate of $\lambda$. Think of this unusual construction as follows: Routing of a generic arrival to a station means that the number of generic customers there is increased by one. Under 
our expanded stationary policy class, denoted by $\mathcal{U}^{\prime}$, a generic arrival may result in an increase by one in the generic customer numbers at any number of stations (from 1 to $N$ ) or that the arrival can simply be ignored, leaving the system state unchanged. This construction allows us, for example, to admit multiple copies of generic arrivals when the system is close to empty and to forbid entry to such arrivals when queue lengths are large. Within $\mathcal{U}^{\prime}$ we will enforce the constraint that the time-average number of stations to which generic arrivals are routed is one.

We write $\widetilde{A}_{n}(u)$ for the time-average generic admission rate at station $n$ under policy $u$ and $\widetilde{R}_{n}(u)=\lambda-\widetilde{A}_{n}(u)$ for the corresponding rejection rate and express the relaxation of (11) by

$$
\bar{C}^{o p t}=\min _{u \in \mathcal{U}^{\prime}} \sum_{n=1}^{N} \widetilde{C}_{n}(u),
$$

where the minimization in (12) is over those $u \in \mathcal{U}^{\prime}$ satisfying

$$
\sum_{n=1}^{N} \widetilde{R}_{n}(u)=(N-1) \lambda .
$$

We also follow Whittle [51] in further relaxing the problem by adopting a Lagrangian approach to the incorporation of the constraint (13) into the objective. We thus obtain a Lagrangian relaxation of (11) expressed by

$$
\widehat{C}(W)=\min _{u \in \mathcal{U}^{\prime}} \sum_{n=1}^{N}\left\{\widetilde{C}_{n}(u)+W \widetilde{R}_{n}(u)\right\}-W(N-1) \lambda .
$$

In (14), $W$ is a Lagrange multiplier that has an economic interpretation as a charge for rejecting a single generic customer. Plainly, we have

$$
\widehat{C}(W) \leq \bar{C}^{\text {opt }} \leq C^{\text {opt }} \text { for all } W \in \mathbb{R} .
$$

We now observe that the nature of the policy class $\mathcal{U}^{\prime}$ and the objective together imply that (14) admits a stationwise decomposition, expressed by

$$
\widehat{C}(W)=\sum_{n=1}^{N} \widehat{C}_{n}(W)-W(N-1) \lambda,
$$

where

$$
\widehat{C}_{n}(W)=\min _{u \in \mathcal{U}}\left\{\widetilde{C}_{n}(u)+W \widetilde{R}_{n}(u)\right\}, \quad 1 \leq n \leq N .
$$

The optimization problem in (15) relates to station $n$ alone, with $\mathcal{U}_{n}$ the class of stationary policies for determining whether to admit (action $a$ ) or reject (action $r$ ) each generic arrival, the goal of optimization being the minimization of an aggregate customer waiting cost $\left(\widetilde{C}_{n}(u)\right)$ and rejection charges $\left(W \widetilde{R}_{n}(u)\right)$. Call this station $n$ 
problem $P_{n}(W)$. The following condition adapts Whittle's notion of indexability to the current problem. It guarantees that the admission control problem $P_{n}(W)$ has a solution which is index based. To express the condition simply we require the notation $a_{n}(u)$ for the set of states (queue lengths) in which the policy $u \in \mathcal{U}_{n}$ admits a generic arrival.

DeFINITION 4.1 (Station Indexability): Station $n$ is indexable if there exists a family of optimal policies for $\left\{P_{n}(W), W \in \mathbb{R}^{+}\right\}$given by $\left\{u_{n, W}, W \in \mathbb{R}^{+}\right\}$such that we have the following:

(i) $a_{n}\left(u_{n, 0}\right)=\emptyset$;

(ii) $a_{n}\left(u_{n, W}\right)$ is increasing in $W$;

(iii) $\forall i \in \mathbb{N}, \exists W \in \mathbb{R}^{+}$such that $i \in a_{n}\left(u_{n, W}\right)$.

The resulting station index $\widetilde{W}_{n}: \mathbb{N} \rightarrow \mathbb{R}^{+}$is given by

$$
\widetilde{W}_{n}(i)=\inf _{W \in \mathbb{R}^{+}}\left\{W ; i \in a_{n}\left(u_{n, W}\right)\right\} \text {. }
$$

The key requirement in Definition 4.1 is part (ii), which requires that as the rejection charge $W$ increases so does the set of queue lengths in which it is optimal to admit a generic arrival. This seems natural. The index $\widetilde{W}_{n}(i)$ may then be thought of as a fair charge for rejecting a customer at station $n$ when the queue length is $i$. Further, if all stations are indexable, it follows that there exists an optimal policy for the Lagrangian relaxation in (14), which, in system state $\boldsymbol{x}=\left(x_{1}, x_{2}, \ldots, x_{N}\right)$, will admit an incoming generic job to each station $n$ whose index $\widetilde{W}_{n}\left(x_{n}\right)$ is no greater than the prevailing charge $W$. It is natural to follow Whittle [51] in proposing as a heuristic for the original problem the policy that routes all incoming generic customers to any station for which this fair charge is the smallest. Formally, when a generic customer arrives, if the system state is $\boldsymbol{x}=\left(x_{1}, x_{2}, \ldots, x_{N}\right)$, then the customer should be routed to any station $n^{*}$ for which

$$
\widetilde{W}_{n^{*}}\left(x_{n^{*}}\right)=\min _{n=1,2, \ldots, N} \widetilde{W}_{n}\left(x_{n}\right) .
$$

We will now proceed to show that, under very mild conditions, the stations are indeed indexable in our model. We will also obtain each station's index in closed form. We now focus exclusively on station $n$ and the corresponding optimization problem $P_{n}(W), W \in \mathbb{R}^{+}$. It will ease notation if, until further notice, we drop the station identifier $n$ from the notation and refer to $P(W), W \in \mathbb{R}^{+}$. In order to deploy the ideas and objects described in Definition 4.1 we need to describe solutions to the $P(W), W \in \mathbb{R}^{+}$. To do that, we develop the sequence $\{W(i), i \in \mathbb{N}\}$ of reals and the corresponding sequence $\{u(i), i \in \mathbb{N}\}$ of monotone admission control policies as 
follows:

$$
\begin{aligned}
W(i) & =\sum_{j=0}^{i} \beta^{j}\left\{\alpha(1-\alpha) \sum_{k=i+1}^{\infty} c(k) \alpha^{k-i-1}+c(i)(1-\alpha)-c(j) \beta\right\}+c(i) \beta^{i+1}, \\
& i \in \mathbb{N},
\end{aligned}
$$

and

$$
u(i) \text { chooses } r \Longleftrightarrow \text { queue length at arrival epoch } \geq i, \quad i \in \mathbb{N} .
$$

It will facilitate the analysis if we make the following technical assumption:

Assumption 4.1: The sequence of expected costs $\{c(i), i \in \mathbb{N}\}$ is nondecreasing such that we have the following:

(i) $c(i) \rightarrow \infty, i \rightarrow \infty$;

(ii) $\sum_{i=0}^{\infty} c(i) \alpha^{i}<\infty$.

Lemma 4.1 is a straightforward consequence of Assumption 4.1.

Lemma 4.1: $\{W(i), i \in \mathbb{N}\}$ is an increasing sequence of positive reals such that $W(i) \rightarrow \infty, i \rightarrow \infty$.

The following key result describes optimal policies for $P(W)$ for all $W \geq 0$. We extend (18) by adopting the notational convention $W(-1)=0$. The proof of Theorem 4.1 will utilize the two following lemmas.

Theorem 4.1: Policy $u(i)$ is optimal for $P(W)$ for $W \in[W(i-1), W(i)), i \in \mathbb{N}$.

Fix $W \in[W(i-1), W(i))$ for some $i \in \mathbb{N}$ and choose $M \in \mathbb{N}$ with $M \geq i+1$. We develop a finite state approximation to $P(W)$ by requiring that generic customers be rejected at queue lengths $M$ and above. Let $P(W, M)$ be the optimization problem that seeks an optimal control under this constraint. We will establish Theorem 4.1 by demonstrating that $u(i)$ is optimal for $P(W, M), W \in[W(i-1), W(i)), M \geq i+1$, and then considering the limit $M \rightarrow \infty$.

Since the control $u(i)$ operates identically whether applied to $P(W)$ or to $P(W, M)$, for $i \leq M-1$, we may write $g(i)$ unambiguously for the long-run average cost rate and $w(i, j)$ for the bias in state $j$ under the operation of policy $u(i), M-1 \geq j \geq 0$. In order to formally define the bias, for fixed $T \in \mathbb{R}^{+}$define $C^{u(i)}(j, T)$ to be the total expected cost incurred by arrivals during the time period $[0, T]$ as the station evolves from initial queue length $j$ under policy $u(i)$. Then the bias in state $j$ under the operation of policy $u(i)$ is given by

$$
w(i, j)=\lim _{T \rightarrow \infty}\left\{C^{u(i)}(j, T)-C^{u(i)}(0, T)\right\}
$$

and is guaranteed to be finite by the ergodicity of the system. 
Lemma 4.2: Control $u(i)$ is optimal for $P(W, M), i \leq M-1$ if

$$
\begin{aligned}
& c(j)+w(i, j+1)-w(i, j) \leq W, \quad 0 \leq j \leq i-1 \\
& \text { and } c(j)+w(i, j+1)-w(i, j) \geq W, \quad M-1 \geq j \geq i \text {. }
\end{aligned}
$$

PROOF: Under action $a$ (accept generic customers) in state $j \geq 1$, possible state transitions are to $j+1$ and $j-1$ with rates $\theta$ and $\mu$, respectively. The corresponding rates under action $r$ are $\eta$ and $\mu$. There are no service completions in state 0. Adopting a uniformization with common transition rate $\theta+\mu$, we develop the average cost optimality equations for $P(W, M)$ as

$$
\begin{aligned}
g^{*}+(\theta+\mu) w^{*}(j)= & \min \left\{\theta c(j)+\theta w^{*}(j+1)+\mu I(j \geq 1) w^{*}(j-1)\right. \\
& +\mu I(j=0) w^{*}(0) ; \eta c(j)+W(\theta-\eta)+\eta w^{*}(j+1) \\
& \left.+(\theta-\eta) w^{*}(j)+\mu I(j \geq 1) w^{*}(j-1)+\mu I(j=0) w^{*}(0)\right\} \\
& \text { for } 0 \leq j \leq M-1,
\end{aligned}
$$

where $g^{*}$ is the optimal long-run average cost, $w^{*}(j)$ is the bias under an optimal policy for initial state $j$, and the indicator function $I(A)=1$ if $A$ is true and 0 otherwise.

Moreover, any stationary policy that takes actions to minimize the right-hand side of (21) for all $0 \leq j \leq M-1$ will be average cost optimal. Hence, policy $u(i)$ will be optimal for $P(W, M)$ if, for $j \leq i-1$,

$$
\begin{aligned}
& \theta c(j)+\theta w(i, j+1)+\mu I(j \geq 1) w(i, j-1)+\mu I(j=0) w(i, 0) \\
& \leq \\
& \quad \eta c(j)+W(\theta-\eta)+\eta w(i, j+1)+(\theta-\eta) w(i, j)+\mu I(j \geq 1) w(i, j-1) \\
& \quad+\mu I(j=0) w(i, 0)
\end{aligned}
$$

with the reverse inequality for $M-1 \geq j \geq i$. The requirements in (20) follow simply. This concludes the proof.

In order to utilize Lemma 4.2, we need the biases $w(i, j), 0 \leq j \leq M-1$. As in the calculations in the paragraphs containing (4) and (5) in Section 3, we deploy an argument based on the fact that the queue length process under $u(i)$ regenerates whenever the queue empties to assert that

$$
w(i, j)=K(i, j)-g(i) T(i, j), \quad 0 \leq j \leq M-1 .
$$

In (23), $K(i, j)$ is the total expected cost incurred (waiting costs and rejection charges) as the queue empties (for the first time) under policy $u(i)$ from an initial position in which $j$ customers are present, and $T(i, j)$ is the corresponding expected time. From (23), it is easy to see that $w(i, 0)=0$. Evaluations of these quantities are given in Lemma 4.3. 


\section{LEMMA 4.3:}

(i) For $i \in \mathbb{Z}^{+}$, we have the following:
(a) $T(i, j)=\sum_{k=0}^{j-1}\left\{\left(1-\beta^{i-1-k}\right)(\mu-\theta)^{-1}+\beta^{i-1-k}(\mu-\eta)^{-1}\right\}$, $0 \leq j \leq i-1$;
(b) $T(i, j)=T(i, i-1)+(j-i+1)(\mu-\eta)^{-1}, i \leq j \leq M-1$;
(c) $K(i, j)=\sum_{k=0}^{j-1}\left\{W(\theta-\eta) \beta^{i-k}(\theta(1-\alpha))^{-1}+\sum_{l=k+1}^{i-1} c(l) \beta^{l-j}\right.$ $\left.+\sum_{l=i}^{\infty} c(l) \beta^{i-k} \alpha^{l-i} \eta \theta^{-1}\right\}, 0 \leq j \leq i-1 ;$
(d) $K(i, j)=K(i, i-1)+\sum_{k=0}^{j-i}\left\{W(\theta-\eta)(\mu-\eta)^{-1}\right.$ $\left.+\sum_{l=k}^{\infty} c(l+i) \alpha^{l+1-k}\right\}, i \leq j \leq M-1$;
(e) $g(i)=K(i, 1)\left\{\theta^{-1}+T(i, 1)\right\}^{-1}$.

(ii) For the case $i=0$, we have the following:

(a) $T(0, j)=j(\mu-\eta)^{-1}, 0 \leq j \leq M-1$;

(b) $K(0, j)=j W(\theta-\eta)(\mu-\eta)^{-1}+\sum_{k=1}^{j} \sum_{l=k}^{\infty} c(l) \alpha^{l-k+1}$, $0 \leq j \leq M-1$;

(c) $g(0)=K(0,1)\left\{\eta^{-1}+T(0,1)\right\}^{-1}$.

ProOF: Choose $i$ and $j$ such that $M-1 \geq j \geq i$. Under policy $u(i)$, the time taken for the queue length to drop from $j$ to $j-1$ (for the first time) is stochastically identical to a busy period of an $M / M / 1$ queue with arrival and service rates $\eta$ and $\mu$, respectively. It follows that

$$
T(i, j)-T(i, j-1)=(\mu-\eta)^{-1}, \quad i \leq j \leq M-1,
$$

and Lemma 4.3(i)(b) follows simply. Further, if $i-1 \geq j \geq 1$, then by conditioning on the time of the first transition after time 0 , we infer that

$$
(\theta+\mu) T(i, j)=1+\theta T(i, j+1)+\mu T(i, j-1), \quad 1 \leq j \leq i-1 .
$$

Combining (25) with (24) and the fact that $T(i, 0)=0$ yields Lemma 4.3(i)(a) simply.

Now, choose $i$ and $j$ such that $M-1 \geq j \geq i$ and consider the system evolving from state $j$ at time 0 under $u(i)$, but where service is abandoned whenever the queue length drops to $j-1$. Hence, whenever the system enters $j-1$, it remains there for an exponentially distributed time with mean $1 / \eta$ before returning to state $j$. An argument based on the fact that the system regenerates upon every entry into $j$ yields the formula

$$
\left\{K(i, j)-K(i, j-1)+W(\theta-\eta) \eta^{-1}+c(j-1)\right\} /\left\{(\mu-\eta)^{-1}+\eta^{-1}\right\}
$$

for the overall cost rate for the system. However, computation of the steady state distribution of the system state yields

$$
W(\theta-\eta)+\sum_{k=j-1}^{\infty} \eta c(k) \alpha^{k+1-j}(1-\alpha)
$$


as an alternative expression. Equating (26) and (27) yields

$$
K(i, j)-K(i, j-1)=W(\theta-\eta)(\mu-\eta)^{-1}+\sum_{k=j}^{\infty} c(k) \alpha^{k-j+1}, \quad i \leq j \leq M-1,
$$

from which Lemma 4.3(i)(d) is easily deduced. Lemma 4.3(i)(c) then follows via a recursion that modifies (25) suitably.

Lemma 4.3(i)(e) is a trivial consequence of the fact that the system evolving under $u(i)$ regenerates upon every entry into state 1 and that the mean time between successive entries is $\theta^{-1}+T(i, 1)$. This completes the proof of Lemma 4.3(i). Part (ii) is dealt with similarly.

With Lemmas 4.2 and 4.3 in place, we now proceed to the proof of Theorem 4.1. Proof OF THEOREM 4.1: Fix $i \in \mathbb{Z}^{+}$. We will show that

$$
c(j)+w(i, j+1)-w(i, j) \geq W, \quad M-1 \geq j \geq i,
$$

where $W \in[W(i-1), W(i))$. Other cases $\left(i \in \mathbb{Z}^{+}, j \leq i-1\right.$, and $\left.i=0\right)$ are dealt with similarly.

First, note that from (23), condition (29) becomes

$$
c(j)+K(i, j+1)-K(i, j)-g(i)\{T(i, j+1)-T(i, j)\} \geq W, \quad M-1 \geq j \geq i .
$$

However, direct calculation based on (18) and Lemma 4.3 yields that, for any $W \in$ $[W(i-1), W(i))$, we have that

$$
g(i) \leq g(j), \quad M-1 \geq j \geq i .
$$

Hence, since $T(i, j+1)-T(i, j) \geq 0$, in order to establish (30) it will be enough to show that

$$
c(j)+K(i, j+1)-K(i, j)-g(j)\{T(i, j+1)-T(i, j)\} \geq W, \quad M-1 \geq j \geq i .
$$

Utilizing Lemma 4.3(i) parts (b), (d), and (e), the requirement in (32) becomes

$$
\begin{aligned}
c(j)+ & W(\theta-\eta)(\mu-\eta)^{-1}+\sum_{k=j+1}^{\infty} c(k) \alpha^{k-j}-(1-\beta)\left\{W(\theta-\eta) \beta^{j}(1-\alpha)^{-1}\right. \\
& \left.+\sum_{k=0}^{j-1} c(k) \beta^{k} \theta+\sum_{k=j}^{\infty} c(k) \alpha^{k-j} \beta^{j} \eta\right\}\left\{\mu-\eta+\beta^{j}(\eta-\theta)\right\}^{-1} \geq W .
\end{aligned}
$$


Now, multiply inequality (33) through by the positive quantity

$$
\Upsilon(\theta, \mu, j)= \begin{cases}\left\{\mu-\eta+\beta^{j}(\eta-\theta)\right\}(\mu-\theta)^{-1}, & \mu \neq \theta \\ 1+j(1-\alpha), & \mu=\theta,\end{cases}
$$

to obtain the inequality

$$
W(j) \geq W
$$

from (18). However, inequality (34) is guaranteed by Lemma 4.1 and the fact that $W \in[W(i-1), W(i))$. Inequality (29) must then follow.

We conclude from Lemma 4.2 that $u(i)$ is optimal for $P(W, M)$ for every $M \geq$ $i-1$. By consideration of the limit $M \rightarrow \infty$, it is straightforward to deduce that $u(i)$ must be optimal for $P(W)$. This concludes the proof.

We now restore the station suffix $n$ and require that Assumption 4.1 hold for each station. Hence, for each $n$ there is a corresponding sequence $\left\{W_{n}(i), i \in \mathbb{N}\right\}$ given by

$$
\begin{aligned}
W_{n}(i)= & \sum_{j=0}^{i} \beta_{n}^{j}\left\{\alpha_{n}\left(1-\alpha_{n}\right) \sum_{k=i+1}^{\infty} c_{n}(k) \alpha_{n}^{k-i-1}+c_{n}(i)\left(1-\alpha_{n}\right)-c_{n}(j) \beta_{n}\right\} \\
& +c_{n}(i) \beta_{n}^{i+1}, \quad i \in \mathbb{N} .
\end{aligned}
$$

There is also a sequence $\left\{u_{n}(i), i \in \mathbb{N}\right\}$ of monotone admission control policies for station $n$ such that

$$
u_{n}(i) \text { chooses } r \Longleftrightarrow \text { queue length at station } n \text { at arrival epoch } \geq i, \quad i \in \mathbb{N} \text {. }
$$

From Theorem 4.1, the policies $\left\{u_{n, W}, W \in \mathbb{R}^{+}\right\}$defined by

$$
u_{n, W}=u_{n}(i), W \in[W(i-1), W(i)), \quad i \in \mathbb{N},
$$

are such that $u_{n, W}$ is optimal for $P_{n}(W), W \in \mathbb{R}^{+}, 1 \leq n \leq N$. Further, we deduce from Lemma 4.1 that the requirements (i)-(iii) of Definition 4.1 are met, with $W_{n}(i)$ the resulting index for station $n$ when the queue length is $i$. These conclusions are summarized in Theorem 4.2.

THEOREM 4.2 (Indexability, Station Index): If station $n$ satisfies Assumption 4.1, then it is indexable with $W_{n}(i)$, the index for queue length $i$.

We conclude that when all stations satisfy Assumption 4.1, the heuristic described in (17), with $W_{n}$ replacing $\widetilde{W}_{n}$ for all $n$, is a natural one for our routing problem. It will be referred to as the Lagrangian relaxation heuristic (LRH) in the remainder of the article. 


\section{EXAMPLES}

In this section, we assume certain structures for the waiting cost function $C_{n}(\cdot)$ and give expressions for the routing indices developed in Sections 3 and 4. The first example considers an increasing non-continuous and non-convex cost function while the second example assumes an increasing, continuous, and strictly convex cost function. In Section 6, we test the performance of our heuristics along with two other heuristics from the literature under the cost structures assumed by these two examples.

\subsection{Example 1: A Noncontinuous and Nonconvex Cost Function}

In this example, we assume that a customer with a system time of $t$ in station $n$ will incur a cost

$$
C_{n}(t)=h_{n} t+d_{n} I\left(t \geq \tau_{n}\right)+\hat{h}_{n}\left(t-\tau_{n}\right)^{+},
$$

where $(y)^{+}=\max (0, y)$. Hence, for each time unit spent in the queue, the customer will incur $h_{n}$. Furthermore, should the customer spend more time in the system than $\tau_{n}$, then she will incur a one-time cost of $d_{n}$ and subsequently an additional cost of $\hat{h}_{n}$ for each additional time unit above $\tau_{n}$. Such cost functions as in (36) are relevant in many settings, including service systems, where customers are given delivery time guarantees, and in emergency response settings, where there is typically a time window (e.g., "golden hour rule"; see, e.g., [35]) for an acceptable level of response time for critically injured patients.

For the cost function given in (36), it can be shown that

$$
c_{n}(i)=\frac{h_{n}(i+1)}{\mu_{n}}+d_{n} \sum_{j=0}^{i} \frac{\left(\mu_{n} \tau_{n}\right)^{j}}{j !} e^{-\mu_{n} \tau_{n}}+\hat{h}_{n} \sum_{j=0}^{i}(i+1-j)\left(\frac{1}{\mu_{n}}\right) \frac{\left(\mu_{n} \tau_{n}\right)^{j}}{j !} e^{-\mu_{n} \tau_{n}}
$$

Then it is easy to see that this cost function trivially satisfies Assumptions 3.1 and 4.1. Moreover, after some tedious but straightforward algebraic manipulations, the policy improvement index (8) can be shown to be

$$
\begin{aligned}
D_{n}(i)= & \frac{h_{n}(i+1)}{\mu_{n}-\Lambda_{n}}+\frac{d_{n} e^{-\mu_{n} \tau_{n}}}{1-\rho_{n}}\left(e^{\Lambda_{n} \tau_{n}}\left(\frac{1-\rho_{n}^{i+1}}{\rho_{n}^{i}}\right)+\sum_{j=0}^{i} \frac{\left(\mu_{n} \tau_{n}\right)^{j}}{j !}-\frac{1}{\rho_{n}^{i}} \sum_{j=0}^{i} \frac{\left(\Lambda_{n} \tau_{n}\right)^{j}}{j !}\right) \\
& +\frac{\hat{h}_{n} e^{-\mu_{n} \tau_{n}}}{\left(1-\rho_{n}\right)^{2} \mu_{n}}\left(\frac{e^{\Lambda_{n} \tau_{n}}\left(1-\rho_{n}^{i+1}\right)}{\rho_{n}^{i}}+\frac{\left(1-\rho_{n}\right)\left(\mu_{n} \tau_{n}\right)^{i+1}}{i !}\right. \\
& \left.+\left(\left(1-\rho_{n}\right)\left(i-\mu_{n} \tau_{n}\right)+1\right) \sum_{j=0}^{i} \frac{\left(\mu_{n} \tau_{n}\right)^{j}}{j !}-\frac{1}{\rho_{n}^{j}} \sum_{j=0}^{i} \frac{\left(\Lambda_{n} \tau_{n}\right)^{j}}{j !}\right) .
\end{aligned}
$$


Similarly, the Lagrangian relaxation index (35) can be shown to have the form

$$
W_{n}(i)=\frac{\left(1-\beta_{n}^{i+1}\right)\left(1-\alpha_{n}\right) R_{n}(i)}{\left(1-\beta_{n}\right) \alpha_{n}^{i}}-S_{n}(i-1),
$$

where

$$
\begin{aligned}
R_{n}(i)= & \frac{h_{n}}{\mu_{n}}\left(\frac{(i+1) \alpha_{n}^{i}-i \alpha^{i+1}}{\left(1-\alpha_{n}\right)^{2}}\right) \\
& +\frac{d_{n} e^{-\mu_{n} \tau_{n}}}{1-\alpha_{n}}\left(e^{\eta_{n} \tau_{n}}+\alpha_{n}^{i} \sum_{j=0}^{i-1} \frac{\left(\mu_{n} \tau_{n}\right)^{j}}{j !}-\sum_{j=0}^{i-1} \frac{\left(\eta_{n} \tau_{n}\right)^{j}}{j !}\right) \\
& +\frac{\hat{h}_{n} e^{-\mu_{n} \tau_{n}}}{\mu_{n}\left(1-\alpha_{n}\right)}\left(\alpha_{n}^{i}\left(i+\left(1 /\left(1-\alpha_{n}\right)\right)-\mu_{n} \tau_{n}\right) \sum_{j=0}^{i-1} \frac{\left(\mu_{n} \tau_{n}\right)^{j}}{j !}\right. \\
& \left.\quad-\frac{1}{1-\alpha_{n}} \sum_{j=0}^{i-1} \frac{\left(\eta_{n} \tau_{n}\right)^{j}}{j !}+\frac{\left(\eta_{n} \tau_{n}\right)^{i}}{(i-1) !}+\frac{e^{\eta_{n} \tau_{n}}}{1-\alpha_{n}}\right)
\end{aligned}
$$

and

$$
\begin{aligned}
S_{n}(i)= & \frac{h_{n} \beta_{n}}{\mu_{n}\left(1-\beta_{n}\right)^{2}}\left(1-(i+2) \beta_{n}^{i+1}+(i+1) \beta_{n}^{i+2}\right) \\
& +\frac{d_{n} e^{-\mu_{n} \tau_{n}}}{1-\beta_{n}}\left(\beta_{n} \sum_{j=0}^{i} \frac{\left(\theta_{n} \tau_{n}\right)^{j}}{j !}-\beta_{n}^{i+2} \sum_{j=0}^{i} \frac{\left(\mu_{n} \tau_{n}\right)^{j}}{j !}\right) \\
+ & \frac{\hat{h}_{n} \beta_{n} e^{-\mu_{n} \tau_{n}}}{\mu_{n}\left(1-\beta_{n}\right)^{2}}\left(\sum_{j=0}^{i} \frac{\left(\theta_{n} \tau_{n}\right)^{j}}{j !}+\beta_{n}^{i}\left((i+1) \beta_{n}^{2}-(i+2) \beta_{n}\right) \sum_{j=0}^{i-1} \frac{\left(\mu_{n} \tau_{n}\right)^{j}}{j !}\right. \\
& \left.+\frac{\left(\theta_{n} \tau_{n}\right)^{i}}{i !}\left((i+1) \beta_{n}^{2}-(i+2) \beta_{n}\right)\right) .
\end{aligned}
$$

\subsection{Example 2: A Continuous and Convex Cost Function}

For this example, we consider a cost function with structural properties that are quite different from those of the cost function of Example 1. To be more precise, we assume that a customer with a system time of $t$ in station $n$ will incur a cost

$$
C_{n}(t)=t^{2}
$$

Note that this cost function is continuous, strictly increasing, and convex. Hence, it satisfies the conditions that are needed for asymptotic optimality of Stolyar's MinDrift rule [40]. 
For this cost function, it can be easily shown that

$$
c_{n}(i)=\frac{(i+1)(i+2)}{\mu_{n}^{2}}
$$

and, hence, Assumptions 3.1 and 4.1 are trivially satisfied. After some algebraic manipulations, the policy improvement index (8) can be shown to be

$$
D_{n}(i)=\frac{(i+1)\left(i\left(1-\rho_{n}\right)+2\right)}{\mu_{n}^{2}\left(1-\rho_{n}\right)^{2}} .
$$

On the other hand, the Lagrangian relaxation index (35) can be shown to have the form

$$
W_{n}(i)=\frac{\left(1-\beta_{n}^{i+1}\right)\left(1-\alpha_{n}\right)}{\left(1-\beta_{n}\right)}\left(\frac{T_{n}(i)}{\alpha_{n}^{i}}+c_{n}(i)\right)-\sum_{j=0}^{i-1} \beta_{n}^{j+1} c_{n}(j),
$$

where

$$
T_{n}(i)=\frac{(i+3)(i+2) \alpha_{n}^{i+1}\left(1-\alpha_{n}\right)^{2}+2 \alpha_{n}^{i+2}\left((i+3)-(i+2) \alpha_{n}\right)}{\left(1-\alpha_{n}\right)^{3} \mu_{n}^{2}} .
$$

\section{NUMERICAL RESULTS}

This section reports our findings from a numerical study that we conducted to assess the performance of the heuristics developed in Sections 3-5 and to observe the effects of some system parameters on the performance of these heuristics. In our numerical study, we consider a system with two service stations $(N=2)$ and assume cost functions in the form of either (36) or (37). For such systems, it is possible to determine optimal routing policies numerically by dynamic programming, thus permitting a complete evaluation of the proposed heuristics.

Our preliminary numerical results suggested that the performance of the heuristics clearly depended on the system load, $\left(\lambda+\eta_{1}+\eta_{2}\right) /\left(\mu_{1}+\mu_{2}\right)$. Therefore, we compared the performance of the heuristics under three different traffic levels:

(i) Light traffic: system load ranges from 0 to 0.6

(ii) Medium traffic: system load ranges from 0.6 to 0.8

(iii) Heavy traffic: system load ranges from 0.8 to 1.0

Under each setting, we generated scenarios by choosing $\eta_{1}, \eta_{2}$, and $\lambda$ uniformly over the interval $[0,1]$ and $\mu_{1}$ and $\mu_{2}$ over the interval $[0,1.5]$ and discarding the cases with system loads that did not fall into the desired range. For each scenario, we computed the performance of the optimal policy, the policy improvement heuristic (PIH), the Lagrangian relaxation heuristic (LRH), the MinDrift heuristic (MDH), the greedy 
heuristic $(\mathrm{GH})$, and the optimal static routing heuristic (SH). Sections 3-5 provide detailed descriptions of how PIH and LRH work. We next explain the MDH, GH, and SH policies.

The MDH, which is due to Stolyar [40], is an index heuristic similar to PIH and LRH. It simply routes each incoming customer to station $n$ for which the corresponding index $M_{n}(i)$ is the smallest when there are $i$ customers at station $n$. For Example 1, the MinDrift index (for station $n$ when there are $i$ customers) is given by

$$
M_{n}(i)= \begin{cases}h_{n} / \mu_{n}, & i / \mu_{n}<\tau_{n} \\ \left(h_{n}+\hat{h}_{n}\right) / \mu_{n}, & i / \mu_{n} \geq \tau_{n},\end{cases}
$$

whereas the MinDrift index for Example 2 is

$$
M_{n}(i)=i / \mu_{n}^{2}
$$

Stolyar [40] proved that the MinDrift heuristic is asymptotically optimal under heavy traffic for strictly convex and continuous waiting cost functions. Therefore, there is no reason to expect that this rule will perform well for Example 1, for which the cost function is neither continuous nor convex. However, both conditions are satisfied in Example 2, and consequently, stronger performance of MDH is expected there, especially so in heavy traffic instances.

The GH is the standard proposal for routing problems. It routes each incoming generic customer to the queue for which the expected cost for that particular customer is the smallest. (Note that GH is equivalent to SDR when cost parameters are the same for all stations.) Finally, the optimal static routing heuristic is the optimal policy among all static routing policies (see (2)) and is the input policy for the second stage of PIH. Note that this heuristic is guaranteed to be inferior to the policy improvement heuristic. However, we still report the performance of SH in order to quantify the improvements gained by state-dependent policies over this static policy.

The remainder of this section is organized as follows. In Section 6.1, we compare the performance of our heuristic policies LRH and PIH with the performances of $\mathrm{MDH}, \mathrm{SH}$, and GH. In Section 6.2, we discuss the effects of two system parameters on the performance of the heuristics under consideration.

\subsection{Comparison of the Heuristic Policies}

In this subsection, we compare the performance of the heuristics for Examples 1 and 2, which are described in Sections 5.1 and 5.2, respectively.

6.1.1. Performance of the heuristics: Example 1. In this part of the study, we assumed a cost function in the form of (36) with the choices $h_{1}=h_{2}=$ $\hat{h}_{1}=\hat{h}_{2}=1, d_{1}=d_{2}=8$, and $\tau_{1}=\tau_{2}=5$. For each traffic setting, we randomly generated 5000 scenarios, and under each scenario, for each heuristic, we computed 
the percentage deviation of the performance from that of the optimal policy and constructed a $99 \%$ confidence interval on the percentage deviation. For each heuristic, we also determined the median, the lower and upper quartiles for the percentage deviation of each policy from the optimal cost, and the number of occasions that heuristic provided the smallest cost among all heuristics considered. We report our findings in Tables 1, 2, and 3 under light, medium, and heavy traffic settings, respectively. Note that the last columns in the tables add up to a value larger than 5000 due to ties among heuristics.

Although LRH, PIH, and GH all perform reasonably well, the numerical results presented in Tables 1-3 demonstrate the superior performance of LRH under each traffic setting. The most important aspect of these tables is the clear evidence it provides that LRH outperforms the standard proposal $\mathrm{GH}$ for the problems studied. One can

TABLE 1. Performance of the Heuristics for Example 1 Under Light Traffic

\begin{tabular}{lcccrr}
\hline Heuristic & $\begin{array}{c}\text { Lower } \\
\text { Quartile }\end{array}$ & Median & $\begin{array}{c}\text { Upper } \\
\text { Quartile }\end{array}$ & $\begin{array}{c}\text { 99\% C.I. } \\
\text { for the Mean }\end{array}$ & \multicolumn{1}{c}{$\begin{array}{c}\text { Best } \\
\text { Heuristic in }\end{array}$} \\
\hline LRH & 0.000 & 0.000 & 0.011 & $0.034 \pm 0.005$ & 4030 scenarios \\
PIH & 0.000 & 0.014 & 0.557 & $0.668 \pm 0.053$ & 1927 scenarios \\
MDH & 0.420 & 8.747 & 42.754 & $31.108 \pm 1.705$ & 223 scenarios \\
GH & 0.018 & 0.327 & 1.597 & $1.224 \pm 0.070$ & 932 scenarios \\
SH & 0.369 & 5.338 & 23.877 & $13.761 \pm 0.628$ & 0 scenarios \\
\hline
\end{tabular}

Note: Numbers except those in the last column are for percentage deviation from the optimal.

Table 2. Performance of the Heuristics for Example 1 Under Medium Traffic

\begin{tabular}{lcrrrr}
\hline Heuristic & $\begin{array}{c}\text { Lower } \\
\text { Quartile }\end{array}$ & Median & $\begin{array}{c}\text { Upper } \\
\text { Quartile }\end{array}$ & $\begin{array}{c}\text { 99\% C.I. } \\
\text { for the Mean }\end{array}$ & \multicolumn{1}{c}{$\begin{array}{c}\text { Best } \\
\text { Heuristic in }\end{array}$} \\
\hline LRH & 0.015 & 0.086 & 0.315 & $0.603 \pm 0.421$ & 3543 scenarios \\
PIH & 0.054 & 0.712 & 2.782 & $2.148 \pm 0.693$ & 1213 scenarios \\
MDH & 3.513 & 22.638 & 64.147 & $43.790 \pm 2.028$ & 73 scenarios \\
GH & 0.416 & 1.653 & 4.018 & $2.685 \pm 0.108$ & 555 scenarios \\
SH & 3.669 & 25.618 & 50.253 & $28.816 \pm 0.900$ & 0 scenarios \\
\hline
\end{tabular}

Note: Numbers except those in the last column are for percentage deviation from the optimal.

Table 3. Performance of the Heuristics for Example 1 Under Heavy Traffic

\begin{tabular}{lcrrrr}
\hline Heuristic & $\begin{array}{c}\text { Lower } \\
\text { Quartile }\end{array}$ & Median & $\begin{array}{c}\text { Upper } \\
\text { Quartile }\end{array}$ & $\begin{array}{c}\text { 99\% C.I. } \\
\text { for the Mean }\end{array}$ & \multicolumn{1}{c}{$\begin{array}{c}\text { Best } \\
\text { Heuristic in }\end{array}$} \\
\hline LRH & 0.735 & 1.728 & 4.799 & $4.230 \pm 0.418$ & 3851 scenarios \\
PIH & 2.433 & 6.130 & 9.912 & $7.880 \pm 0.361$ & 720 scenarios \\
MDH & 5.695 & 14.040 & 32.616 & $25.603 \pm 1.235$ & 414 scenarios \\
GH & 2.450 & 4.898 & 8.361 & $5.782 \pm 0.150$ & 528 scenarios \\
SH & 13.885 & 29.998 & 43.946 & $29.514 \pm 0.689$ & 0 scenarios \\
\hline
\end{tabular}

Note: Numbers except those in the last column are for percentage deviation from the optimal. 
also observe from these tables that the performance of all heuristics except for MDH deteriorates as the system load increases. Note, however, that the mean performance of LRH is around $4.23 \%$ above optimal, with median $1.728 \%$ even under heavy traffic. Also, comparison of the median and quartiles with the mean performance indicates that the distribution of the performances are skewed to the right for all the heuristics. The performance of MDH is clearly poor; however, this is not surprising since MDH was developed under assumptions that are not satisfied for the cost function considered for this example. One observation is that MDH seems to provide its best performance under heavy traffic and its worst performance under medium traffic.

Although LRH clearly seems to be the best heuristic, comparison of PIH and GH is more complicated. PIH seems to be the heuristic that is affected most by the increase in the traffic. Its performance gets significantly worse as we move from medium traffic to heavy traffic. Under heavy traffic, the mean performance of GH is better than that of PIH. However, PIH is the best heuristic in more scenarios than is GH. At other traffic levels, PIH outperforms GH in all aspects.

Finally, Tables 1-3 clearly show that state-dependent policies provide substantial improvements over the "best" static policy, SH. However, these improvements obviously come at the expense of keeping the system state information at all times (or at least observing the queues when routing the generic customers).

6.1.2. Performance of the heuristics: Example 2. In this subsection, we assume that the waiting cost function is in the form of (37). Note that this cost function satisfies all of the assumptions needed for the asymptotic optimality of MDH (see Stolyar [40]) and, therefore, MDH is expected to perform well, at least under heavy traffic.

For each traffic setting, we randomly generated 5000 scenarios and computed the performance of each heuristic under each scenario. Tables 4, 5, and 6 present our findings under light, medium, and heavy traffic, respectively. Similar to our results for Example 1, LRH has the best performance among all of the heuristics considered under this convex and continuous function. Except for MDH, the performance of all the heuristics worsen as the traffic intensity increases. The effect of the traffic load on MDH is not clear, since one would reach different conclusions depending on

Table 4. Performance of the Heuristics for Example 2 Under Light Traffic

\begin{tabular}{lcccrr}
\hline Heuristic & $\begin{array}{c}\text { Lower } \\
\text { Quartile }\end{array}$ & Median & $\begin{array}{c}\text { Upper } \\
\text { Quartile }\end{array}$ & $\begin{array}{c}\text { 99\% C.I. } \\
\text { for the Mean }\end{array}$ & \multicolumn{1}{c}{$\begin{array}{c}\text { Best } \\
\text { Heuristic in }\end{array}$} \\
\hline LRH & 0.000 & 0.000 & 0.011 & $0.046 \pm 0.007$ & 4038 scenarios \\
PIH & 0.000 & 0.017 & 0.706 & $0.915 \pm 0.074$ & 1821 scenarios \\
MDH & 0.698 & 3.048 & 7.809 & $8.116 \pm 0.874$ & 192 scenarios \\
GH & 0.028 & 0.505 & 2.260 & $1.700 \pm 0.095$ & 836 scenarios \\
SH & 0.394 & 6.543 & 35.792 & $20.142 \pm 0.943$ & 0 scenarios \\
\hline
\end{tabular}

Note: Numbers except those in the last column are for percentage deviation from the optimal. 
TABLE 5. Performance of the Heuristics for Example 2 Under Medium Traffic

\begin{tabular}{lccrrr}
\hline Heuristic & $\begin{array}{c}\text { Lower } \\
\text { Quartile }\end{array}$ & Median & $\begin{array}{c}\text { Upper } \\
\text { Quartile }\end{array}$ & $\begin{array}{c}\text { 99\% C.I. } \\
\text { for the Mean }\end{array}$ & \multicolumn{1}{c}{$\begin{array}{c}\text { Best } \\
\text { Heuristic in }\end{array}$} \\
\hline LRH & 0.017 & 0.130 & 0.442 & $0.339 \pm 0.018$ & 3660 scenarios \\
PIH & 0.051 & 1.000 & 4.094 & $2.827 \pm 0.284$ & 1134 scenarios \\
MDH & 1.770 & 4.503 & 8.599 & $6.408 \pm 0.360$ & 149 scenarios \\
GH & 0.679 & 2.525 & 5.778 & $3.832 \pm 0.145$ & 472 scenarios \\
SH & 3.735 & 39.943 & 83.889 & $47.099 \pm 1.567$ & 0 scenarios \\
\hline
\end{tabular}

Note: Numbers except those in the last column are for percentage deviation from the optimal.

Table 6. Performance of the Heuristics for Example 2 Under Heavy Traffic

\begin{tabular}{lrrrrr}
\hline Heuristic & $\begin{array}{c}\text { Lower } \\
\text { Quartile }\end{array}$ & Median & $\begin{array}{c}\text { Upper } \\
\text { Quartile }\end{array}$ & $\begin{array}{c}\text { 99\% C.I. } \\
\text { for the Mean }\end{array}$ & \multicolumn{1}{c}{$\begin{array}{c}\text { Best } \\
\text { Heuristic in }\end{array}$} \\
\hline LRH & 1.302 & 2.588 & 6.597 & $6.004 \pm 0.328$ & 2849 scenarios \\
PIH & 3.315 & 9.248 & 15.469 & $18.871 \pm 1.845$ & 982 scenarios \\
MDH & 2.634 & 5.388 & 10.173 & $7.276 \pm 0.225$ & 1193 scenarios \\
GH & 4.016 & 7.131 & 11.053 & $7.951 \pm 0.188$ & 224 scenarios \\
SH & 22.539 & 58.985 & 92.626 & $61.173 \pm 1.759$ & 0 scenarios \\
\hline
\end{tabular}

Note: Numbers except those in the last column are for percentage deviation from the optimal.

whether the mean and the confidence interval or median and the quartiles are chosen to be the deciding performance measure. However, if compared with the performance of all the other heuristics at all traffic levels, one can observe that, as expected, the relative performance of MDH is the best when under heavy traffic. However, LRH still seems to perform slightly better than MDH even under heavy traffic (as defined in this article). PIH performs relatively well under light and medium traffic, but under heavy traffic, its overall performance is poor. On the other hand, GH is not as good as PIH under light and medium traffic, but its performance under heavy traffic is much better than that of PIH on the average. However, even under heavy traffic, PIH is the best heuristic in more scenarios than GH is. Finally, as in Example 1, the performance of SH is clearly poor, demonstrating the superiority of state-dependent policies.

\subsection{Effects of System Parameters on the Performance of the Heuristics}

In this subsection, we focus our attention on Example 1, assuming a cost function in the form of (36) with parameter values set as in Section 6.1.1, and investigate the effects of two system parameters on the performances of $\mathrm{LRH}, \mathrm{PIH}$, and GH, the three best heuristics for this example.

- Effects of dedicated load heterogeneity: In the system under consideration, each service station has its own dedicated customers and therefore has its own capacity that can be allocated to the service of generic customers. If these residual capacities for the two stations are close to each other, we say 
that the dedicated loads are homogeneous; otherwise we say that they are heterogeneous. To observe the effects of the heterogeneity of the stations on the performance of the heuristics, we first define a heterogeneity index, $\Phi$, as

$$
\Phi=\left|1-\frac{\eta_{1} / \mu_{1}}{\eta_{2} / \mu_{2}}\right| .
$$

After computing the heterogeneity index for each of the 5000 scenarios, we ordered all scenarios according to this index from the smallest to the largest. Then we computed the moving average (of order 2000) of the mean performance across the 5000 scenarios (see Fig. 1 for plots of the moving averages). In all cases, under each traffic setting, we observed a worsening performance of the heuristics with an increase in $\Phi$. Hence, the more balanced the system load is across the two stations, the better is the average performance of all the heuristics. However, the deterioration in performance is more significant for GH, less so for LRH, and even less so for PIH. Furthermore, for LRH and $\mathrm{PIH}$, under heavy traffic, the average performance does not seem to change drastically as we go from very low levels of heterogeneity to mid levels,
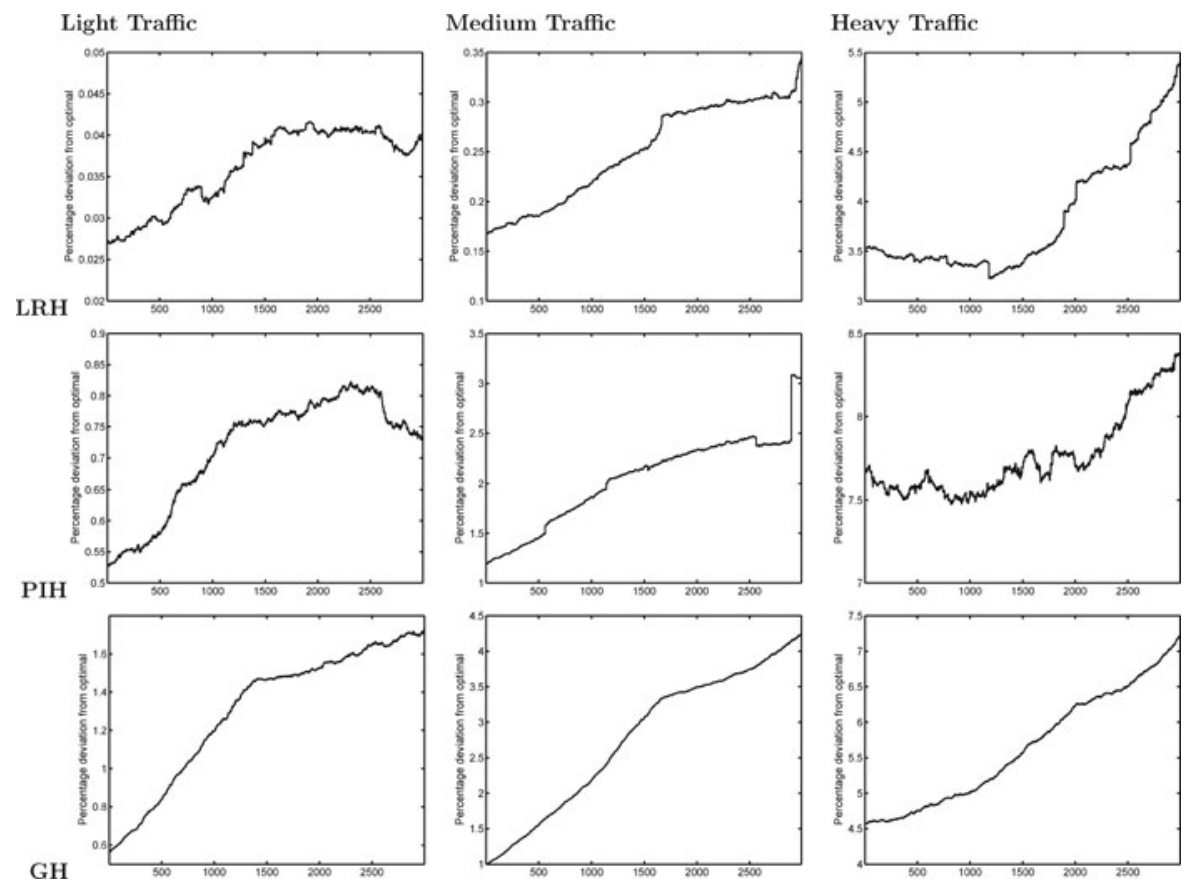

FiguRE 1. Moving average plots of percentage deviations of heuristics from the optimal with respect to the number of scenarios that are ordered according to the increasing heterogeneity index of dedicated load $\Phi$. 
whereas the performance starts to deteriorate significantly as we go from mid levels to high levels. On the other hand, the performance of GH worsens at a seemingly constant level as we go from very low levels of heterogeneity to very high levels.

- Effects of dedicated arrival rates: We have also studied the numerical results to identify cases in which the heuristic algorithms performed poorly. We observed that the dedicated arrival rates have an interesting effect on the performance of the heuristics. To observe this effect, we ordered the scenarios with respect to their total dedicated arrival rates, $\eta_{1}+\eta_{2}$, and carried out an analysis similar to that for the load heterogeneity (see Fig. 2). We observed that the smaller the total dedicated arrival rate, the worse is the performance of the LRH on the average. (Note, however, that LRH still has the best performance among all the heuristics even over the region where its performance is poor.) Its performance is significantly worse for very low levels of the dedicated arrival rate. This is surprising since lower dedicated arrival rates indicate low traffic intensity, which overall seems to have a positive impact on the performance of all heuristics.
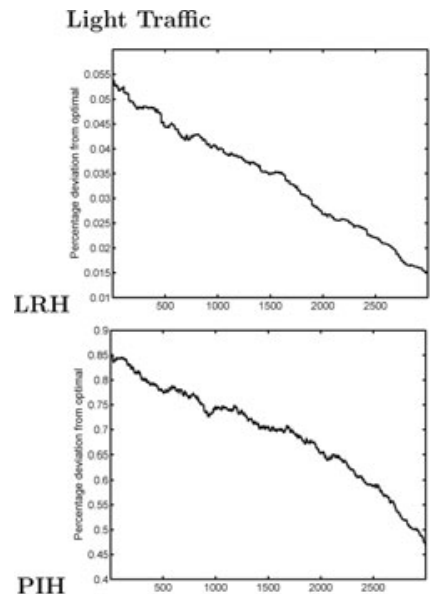

PIH

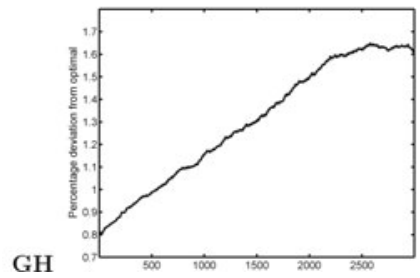

Medium Traffic
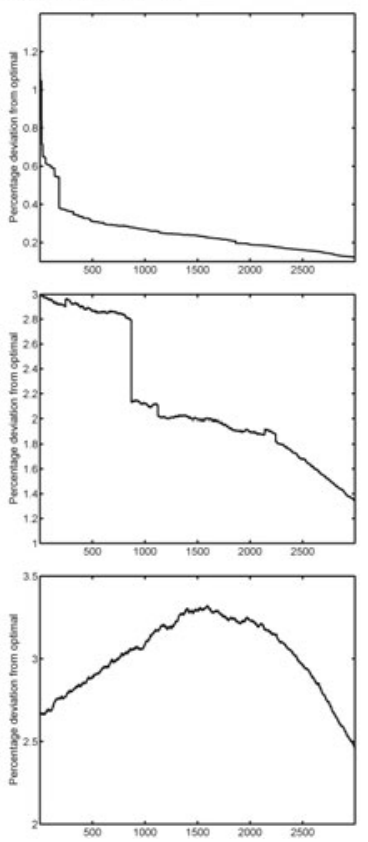

Heavy Traffic
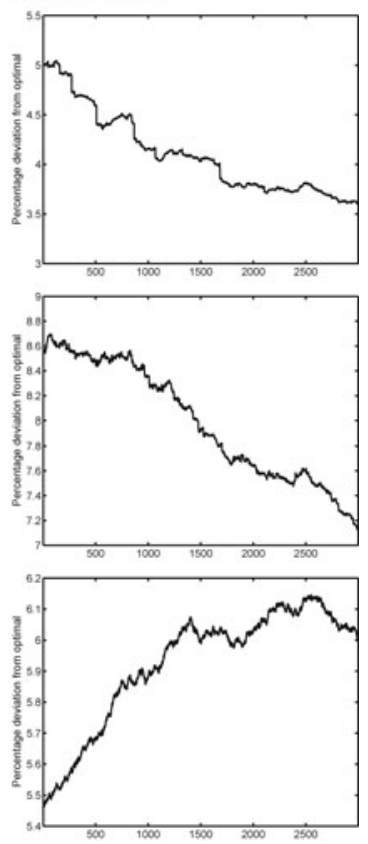

Figure 2. Moving average plots of percentage deviations of heuristics from the optimal with respect to the number of scenarios that are ordered according to the increasing total dedicated arrival rates $\eta_{1}+\eta_{2}$. 
We observed a similar effect on the performance of PIH although the effect does not seem to be as strong as for LRH. On the other hand, the effect on GH seems to depend on the total traffic load on the system. Under medium traffic, very low and very high levels of dedicated traffic rate have a positive effect on the performance, whereas moderate levels yield worse performance on the average. Under light and heavy traffic, the performance worsens as the total dedicated arrival rate increases.

\section{CONCLUSIONS}

For realistically sized routing problems that can be modeled as Markov decision problems, standard solution approaches based on direct application of stochastic DP are not practical. The conventional proposal is to adopt a greedy heuristic $(\mathrm{GH})$, which is sometimes called an individually optimal policy for routing. In the context of a general model for waiting costs that accommodates dedicated traffic at each station, we developed two alternative heuristics, based respectively on DP policy improvement (PIH) and Lagrangian relaxation (LRH). The theoretical challenge with regard to the latter heuristic concerns the demonstration that station indexability is available at this level of generality. A numerical study makes clear that LRH performs very closely to the optimal policy and offers a considerable improvement over $\mathrm{GH}$ at all traffic levels. It also performs better than the MinDrift heuristic (MDH), which is known to be asymptotically optimal under certain conditions.

In this article, we considered scenarios in which the waiting cost for a customer possibly depends on the server. Although there are various examples where this generalization is relevant (e.g., in the case of the routing problem that a manufacturer faces in outsourcing warranty repairs to different vendors that charge different costs; see [36]), an interesting extension would be to also allow waiting cost for a customer to depend on the customer's type: dedicated or generic. In fact, PIH as described in this model can easily be extended accordingly. However, this is not the case for LRH since establishing indexability appears to be a significant challenge when waiting costs depend on the customer type. Another direction for future research would be to consider multiple types of generic customers each differing in their waiting costs. Such a generalization would be of particular interest to call centers that serve a heterogeneous group of users and seek ways of providing a better service for their more "valuable" customers. It would also be of interest in health care operations, for which patients have significantly different waiting cost structures depending on their health conditions.

\section{Acknowledgments}

The research of the first author was supported by the National Science Foundation under grant CMMI0715020. The second author acknowledges support given by Edinburgh University and the British Council during her doctoral studies. The third author acknowledges EPSRC support made available through grant GR/S45188/01. The research of the fourth author was supported by the National Science Foundation under grant CMMI-0620736. 


\section{References}

1. Ansell, P.S., Glazebrook, K.D. \& Kirkbride, C. (2003). Generalised "join the shortest queue" policies for the dynamic routing of jobs to multi-class queues. Journal of the Operational Research Society 54: 379-389.

2. Ansell, P.S., Glazebrook, K.D., Niño-Mora, J. \& O’Keeffe, M. (2003). Whittle's index policy for a multi-class queueing system with convex holding costs. Mathematical Methods in Operations Research 57: 21-39.

3. Armony, M. (2005). Dynamic routing in large-scale service systems with heterogeneous servers. Queueing Systems 51: 287-329.

4. Armony, M. \& Maglaras, C. (2004). On customer contact centers with a call-back option: Customer decisions, routing rules, and system design. Operations Research 52: 271-292.

5. Armony, M. \& Maglaras, C. (2004). Contact centers with a call-back option and real time delay information. Operations Research 52: 527-545.

6. Bassamboo, A., Harrison, J.M. \& Zeevi, A. (2005). Dynamic routing and admission control in highvolume service systems: Asymptotic analysis via multi-scale fluid limits. Queueing Systems 51: 249-285.

7. Becker, K.J., Gaver, D.P., Glazebrook, K.D., Jacobs, P.A. \& Lawphongpanich, S. (2000). Allocation of tasks to specialised processors: a planning approach. European Journal of Operational Research 126: 80-88.

8. Bhulai, S. (2004). Dynamic routing policies for multi-skill call centers. Technical report 2004-11, Vrije University, Amsterdam.

9. Bhulai, S. \& Koole, G. (2003). On the structure of value functions for threshold policies in queueing models. Journal of Applied Probability 40: 613-622.

10. Charnsirisakskul, K., Griffin, P.M. \& Keskinocak, P. (2004). Order selection and scheduling with leadtime flexibility. IIE Transactions 36: 697-707.

11. Foley, R.D. \& McDonald, D.R. (2001). Join the shortest queue: stability and exact asymptotics. Annals of Applied Probability 11: 569-607.

12. Foschini, G.J. \& Salz, J. (1978). A basic dynamic routing problem and diffusion. IEEE Transactions on Communications 26: 320-327.

13. Gans, N. \& Zhou, Y.P. (2003). A call routing problem with service level constraints. Operations Research 51: 255-271.

14. Glazebrook, K.D., Lumley, R.R. \& Ansell, P.S. (2003). Index heuristics for multiclass $M / G / 1$ systems with nonpreemptive service and convex holding costs. Queueing Systems 45: 81-111.

15. Glazebrook, K.D., Kirkbride, C. \& Ouenniche, J. (2009). Index policies for the admission control and routing of impatient customers to heterogeneous service stations. Operations Research. In press.

16. Glazebrook, K.D., Mitchell, H.M. \& Ansell, P.S. (2005). Index policies for the maintenance of a collection of machines by a set of repairmen. European Journal of Operational Research 165: 267-284.

17. Glazebrook, K.D., Niño-Mora, J. \& Ansell, P.S. (2002). Index policies for a class of discounted restless bandits. Advances in Applied Probability 34: 754-774.

18. Gross, D. \& Harris, C.M. (1998). Fundamentals of queueing theory. New York: Wiley.

19. Harrison, J.M. \& Zeevi, A. (2004). Dynamic scheduling of a multi-class queue in the Halfin-Whitt heavy traffic regime. Operations Research 52: 243-257.

20. Hopp, W.J. \& Sturgis, M.L.R. (2000). Quoting manufacturing due dates subject to a service level constraint. IIE Transactions 32: 771-784.

21. Hordijk, A. \& Koole, G. (1990). On the optimality of the generalized shortest queue policy. Probability in the Engineering and Information Sciences 4: 477-487.

22. Houck, D.J. (1987). Comparison of policies for routing customers to parallel queueing systems. Operations Research 35: 306-310.

23. Johri, P.K. (1989). Optimality of the shortest line discipline with state-dependent service rates. European Journal of Operational Research 41: 157-161.

24. Jones, D. (2004). Some hospital ERs begin guaranteeing quick service. USA Today, December 3. 
25. Kelly, F.P. \& Laws, C.N. (1993). Dynamic routing in open queueing networks: Brownian models, cut constraints and resource pooling. Queueing Systems 13: 47-86.

26. Koole, G., Sparaggis, P.D. \& Towsley, D. (1999). Minimizing response times and queue lengths in systems of parallel queues. Journal of Applied Probability 36: 1185-1193.

27. Krishnan, K.R. (1987). Joining the right queue: A Markov decision rule. In Proceedings of the 26th IEEE Conference on Decision Control, pp. 1863-1868.

28. Krishnan, K.R. (1990). Joining the right queue: A state-dependent decision rule. IEEE Transactions on Automatic Control 35: 104-108.

29. Krishnan, K.R. \& Ott, T.J. (1986). State-dependent routing for telephone traffic: Theory and results. In Proceedings of the 25th IEEE Conference on Decision Control, pp. 2124-2128.

30. Laws, C.N. (1992). Resource pooling in queueing networks with dynamic routing. Advances in Applied Probability 24: 699-726.

31. MacKenzie, E.J., Hoyt, D.B., Sacra, J.C., Jurkovich, G.J., Carlini, A.R., Taitelbaum, S.D. \& Teter, H., Jr. (2003). National inventory of hospital trauma centers. Journal of the American Medical Association 289: $1515-1522$.

32. Mandelbaum, A. \& Stolyar, A.L. (2004). Scheduling flexible servers with convex delay costs: Heavy traffic optimality of generalized $c \mu$-rule. Operations Research 52: 836-855.

33. Menich, B. \& Serfozo, R. (1991). Optimality of routing and servicing in dependent parallel processing systems. Queueing Systems 9: 403-418.

34. Niño-Mora, J. (2002). Dynamic allocation indices for restless projects and queueing admission control. Mathematical Programming 93: 361-413.

35. Osterwalder, J.J. (2002). Can the "golden hour of shock" be safely extended in blunt polytrauma patients. Prehospital and Disaster Medicine 17: 75-80.

36. Opp, M., Glazebrook, K. \& Kulkarni, V. (2005). Outsourcing warranty repairs: Dynamic allocation. Naval Research Logistics 52: 381-398.

37. Ross, K.W. \& Yao, D.D. (1991). Optimal load balancing and scheduling in a distributed computer system. Journal of the Association for Computing Machinery 38: 676-690.

38. Sassen, S.A.E., Tijms, H.C. \& Nobel, R.D. (1997). A heuristic rule for routing customers to parallel servers. Statistica Neerlandica 51: 107-121.

39. Sparaggis, P.D., Towsley, D. \& Cassandras, C.G. (1993). Extremal properties of the SNQ and the LNQ policies in finite capacity systems with state-dependent service rates. Journal of Applied Probability 30: 223-236.

40. Stolyar, A.L. (2005). Optimal routing in output-queued flexible server systems. Probability in the Engineering and Informational Sciences 19: 141-189.

41. Teh, Y. \& Ward, A. (2002). Critical thresholds for dynamic routing in queueing networks. Queueing Systems 42: 297-316.

42. Tezcan, T. (2005). Optimal control of distributed parallel server systems under the Halfin and Whitt regime. Mathematics of Operations Research 33: 51-90.

43. Tijms, H.C. (1994). Stochastic models: An algorithmic approach. New York: Wiley.

44. Towsley, D., Sparaggis, P.D. \& Cassandras, C.G. (1993). Optimal routing and buffer allocation for a class of finite capacity queueing systems. IEEE Transactions on Automatic Control 37: 1446-1451.

45. Van Mieghem, J.A. (1995). Dynamic scheduling with convex delay costs: The generalized $c \mu$ rule. The Annals of Applied Probability 5: 809-833.

46. Wallace, R.B. \& Whitt, W. (2005). A staffing algorithm for call centers with skill-based routing. Manufacturing and Service Operations Management 7: 276-294.

47. Weber, R. (1978). On the optimal assignment of customers to parallel servers. Journal of Applied Probability 15: 406-413.

48. Wein, L.M. (1991). Brownian networks with discretionary routing. Operations Research 39: 322-340.

49. Whitt, W. (1986). Deciding which queue to join: Some counterexamples. Operations Research 34: $55-62$. 
50. Whittle, P. (1996). Optimal control: Basics and beyond. New York: Wiley.

51. Whittle, P. (1988). Restless bandits: Activity allocation in a changing world. Journal of Applied Probability A25: 287-298.

52. Winston, W. (1977). Optimality of the shortest-processing-time discipline. Journal of Applied Probability 14: 181-189. 